\title{
Application and Prospect of Softswitch in Power Communication System
}

\author{
Xiaobei Wang, Jinxiang Cheng, Shiwen Chen, Peng Chen and Jinzhi Guo
}

State Grid Hubei Electric Power Company Huanglongtan Hydropower Plant, Shiyan 442000, China

Keywords: Softswitch, power communication system, power plant, application.

\begin{abstract}
With the power system to achieve "plant network separation", soft switching technology in the application of power plants more and more attention. At present, part of the power grid, transmission network equipment, fiber optic cable prices cheaper, more and more wide transmission bandwidth. For power generation enterprises, there are transmission equipment within the power plant coverage of the site, temporarily will not change the existing circuit-switched mode. For the power generation enterprises in a very large end node (plant substation voice) has not yet been resolved, soft switching technology can effectively solve the power supply voice switching problems, resulting in more economic benefits, cost savings.
\end{abstract}

\section{Background}

Softswitch is the control function entity of the next generation network, which provides the call control and connection control function for the next generation network with real-time service. It is the core of the next generation network call and control. In short, the softswitch is the entity that implements the "call control" function of the traditional PBX, but the traditional "call control" function is combined with the business, and the call control functions required by different services are different, which requires softswitch the provision of call control functions is the basic call control for various services. The basic meaning of softswitch is to separate the call control function from the media gateway. The basic call control function is realized through the software on the server or the network element, including call routing, management control, connection control (establishment session, dismantling session), letter So that interoperability (such as signaling from the 7 to IP signaling) and so on[4]. This separation establishes a separate plane for control, switching, and software-programmable functions that enable service providers to freely combine transport services with control protocols to achieve business transfer. All protocols for softswitch are IP-based, and they have the openness and flexibility of all IP-based protocols. Most importantly, the softswitch uses an open application programming interface (API) that simplifies the complexity of the signaling structure and control, and has openness to network technology, access technology, and intelligent services. Allowing the introduction of new services in the exchange mechanism, and the original four switches (long distance, tandem switch), 5 switches (local switches) can still be retained through the SS7 link [5].

\section{Softswitch Network Structure Technology}

\subsection{Softswitch Network Structure}

Power network to softswitch as the core of the network has three characteristics: First, the use of an open network architecture system, the traditional switch function modules into separate network components, the user can be based on business needs free combination of functional parts Set up the network, the interface between the components of the standardization of the interface can achieve a variety of heterogeneous network interoperability. The second is based on the business-driven network, its function is the separation of business and call control, call and bearer separation, so that business is truly independent of the network, flexible and efficient implementation of the business, the user can configure and define their own business characteristics [6], Concerned about the network form of the bearer service and the type of terminal, so that the provision of business and application has greater flexibility. Third, based on a unified protocol and packet-based network, because the 
existing information network, whether it is telecommunications network, computer network or cable network can not be one of the network-based platform to grow information infrastructure [7], with IP Development, people have recognized that the telecommunications network, computer networks and cable television network will eventually pool to a unified IP network, which is commonly referred to as "triple play" integration trend [8].

The location of the softswitch in the network is divided into media / access layer, transport layer, control layer and service / application layer. The functions of each layer are as follows [9].

(1) media / access layer: to provide a variety of user terminals, user premises network and traditional communication network access to the core network gateway.

(2) transport layer: for a variety of media to provide broadband transmission channel, and the information routing to the destination.

(3) control layer: the main to provide call control, processing functions and protocol functions, business functions and interoperability.

(4) business / application layer: the use of the underlying resources to provide users with a variety of network services and resource management.

\subsection{External Interface of Softswitch}

Softswitch as an open entity, with an external interface must use an open protocol, various interfaces and their use of the protocol as follows.

(1) interface between the media gateway (MG) and the softswitch: the media gateway is used for softswitch to carry control, resource control and management of the media gateway. This interface can use the Media Gateway Control Protocol (MGCP), IP Device Control Protocol (IPDC), or H.248 protocol [10].

(2) interface between the signaling gateway (SG) and the softswitch: The signaling gateway is used to transmit the signaling information between the softswitch and the media gateway. This interface can use Signaling Control Transmission Protocol (SCTP) or other similar protocols.

(3) interface between softswitch: to achieve the interaction between different softswitch. This interface can use the Session Initiation Protocol (SIP-T) or H.323 protocol.

(4) interface between softswitch and application / service: provide access to various databases, tripartite application platform, various functions of the server interface, to achieve value-added services, management services and tripartite application support. Such as: Softswitch and application server interface, you can use the SIP protocol or API (such as Parlay), to provide a tripartite application and a variety of value-added services support functions; Softswitch and policy server interface, you can use the COPS protocol, The implementation of the network equipment to work on the dynamic intervention; Softswitch and network management center interface, you can use the SNMP protocol to achieve network management; · Softswitch and intelligent network service control point (SCP) interface, you can use the INAP protocol, To achieve the existing intelligent network business support.

\section{Softswitch Technology Advantages}

Compared with the traditional switch, the softswitch technology has the following major advantages:

(1) soft switching technology has a lower cost, in the use of the process, the use of open platform and ordinary computer, it is easy to carry out And in the whole process, the price is very cheap, has a greater advantage, and third parties can also easily use the appropriate soft switching equipment, in this case, you can in the corresponding softswitch platform Use the network new business.

(2) the user's choice has a diversity. The traditional switch in the use of the process, generally by a business to provide the appropriate software and hardware maintenance and development, system updates and all other business, so that makes the user's choice of space significantly reduced, making the user did not The corresponding initiative, especially in the follow-up maintenance costs and no corresponding options, but the softswitch technology can solve this problem, the use of softswitch 
technology, you can establish a unified, standard onion space, And in the use of the process, the user can be independent of the supplier's choice, for the application of the software is extremely beneficial.

(3) has a high reliability. Users in the process of network group, the use of soft switching technology can be fully functional software classification, all assigned to the corresponding backbone network, in the process, mainly based on the computer as a platform for system programming Processing, so that the network will become more secure and reliable.

\section{Softswitch Technology in the Application of Softswitch in Power Generation Enterprises}

At present, most companies are moving from the traditional stage to the converged network stage transition, some leading enterprises have begun to transition to the integration of communication stage. Converged networks can make it easier for organizations to add and deploy new features to increase user productivity.

Power generation enterprises under the various power plants are widely distributed, the number of more, and "plant network separation", the power generation companies do not have their own transmission network. Therefore, the power generation company is different from the grid company, communication status and the ordinary enterprise network is very similar. In the future, with the popularity of various application systems, power generation companies may rent their own power or telecommunications bandwidth to set up their own dedicated data network. If in order to solve the voice of each power plant and then go to rent their circuit resources, virtually increased a lot of costs.

Power communication network is one of the most widely distributed networks in the world, including fiber, microwave, carrier and other transmission media. Which formed a fiber network, microwave and other forms of network, a variety of networks have their own exchange equipment, multiple access equipment, interconnection between these networks there is a big difficulty. If the information needs to be transmitted between different media networks, more conversion sessions will be needed. This not only caused the waste of resources, but also the management of the entire power communication network has also brought great inconvenience. The introduction of softswitch technology will solve the following problems:

\section{(1) Network Communication in Power Communication Network.}

The telephone network is a kind of switching network, and has the unique carrier system of power system; at the same time there are also computer networks in power communication networks, which are IP-based packet networks. Softswitch can provide a variety of signaling protocols to support the interface, can be very good to achieve the telephone network and the computer network between the signaling interoperability and interoperability problems of different gateways. This makes it easier for the computer network to manage and support the telephone network. The telephone network can also be used in conjunction with the computer network to provide better service.

\section{(2) In Order to Improve the Application of Quality of Service}

At present, the information transmitted in the power communication network is mainly voice and data, but with the evolution of the network and the continuous development of computer technology, the video business and multimedia business also put forward new requirements. Softswitch technology not only supports voice services, the use of new network facilities can provide a variety of value-added services and supplementary services, and softswitch provides an open application program interface (API), very easy to provide new business. This is the most popular power system call center (also known as customer service center), the introduction of soft switching technology is undoubtedly a wise choice, based on softswitch call center can use lower cost, shorter period Users to provide better service, and better establish the image of the power system.

\section{(3) Unified the Application of Different Media.}

Power communication network in a variety of transmission media, and each of the more independent, have their own set of equipment, if the introduction of soft switching technology, in a switching server can be a variety of media information exchange. This not only in the economic aspects of the equipment to avoid the waste, but also improve the reliability of the network, a variety of media networks to achieve a certain degree of integration of interoperability in different media 
networks to transfer information also eliminates the complex conversion links. In the management is also more convenient, just a device can be maintained to achieve the entire network of information exchange.

(4) Other Aspects of the Function Softswitch with Operation and Maintenance Functions.

For the complex power system, the introduction of softswitch can be unified on a variety of business statistics. If the fault can also be issued in a timely manner alarm signal. In addition, the soft exchange can also collect a detailed list of electricity and telephone charges to achieve the billing. Softswitch technology is a new technology, its application will not be limited to the above aspects. The introduction of softswitch in the power communication network will produce many aspects of the effect.

\section{Concluding Remarks}

With the rapid development of power transmission network technology, the evolution of the traditional circuit-switched network to the next generation network is unstoppable, and the softswitch will be the key technology of the next generation network. Its appearance is taken in the openness and programmability of the network. The first step, on behalf of the direction of the development of the network, will make a great contribution to the evolution of the network, not only in the public communications network, in all kinds of private networks, including power dedicated communications networks have application prospects, but the softswitch is only the network revolution Prelude, there are many problems need to be further explored, soft switching technology is constantly improving and developing.

\section{References}

[1] Xing Yanxia. et al. Softswitch technology and network convergence . Mobile Communications, 2005, Vol. 29 (6).

[2] Liu Weihong.et al. Softswitch technology in power communication network. Zhejiang University, 2008.

[3] Guo Jinghong, Cai Bin, Gao Yun, et al. Next generation network and its power development strategy during the "Eleventh Five-Year" period. Power System Communications, 2006, Vol. 27 (10).

[4] Qiang Lei.et al. Softswitch based on the next generation network networking technology. Beijing: People's Posts and Telecommunications Press, 2005.

[5] Peng Qingqing, Xiang Li, Lu Changyan, et al. National Electric Power Dispatching Data Network. Power System Automation, 2004, Vol. 28 (8).

[6] Gao Longhua, Wang Li.et al. Application of Softswitch Technology in Power Communication Network. Modern Communications, 2003, Vol. (5).

[7] Li Wenyaoet al. Study on Functional Structure Model of Next Generation Network. China Journal of Data Communications, 2003, Vol. (3).

[8] Wang Jun, Zhang Jianwu.et al. PSTN network and NGN network interoperability. Modern Communications, 2003, Vol. (3).

[9] Chalie.et al. Research on the Combination of Softswitch Network and Existing Network. JOURNAL OF BEIJING UNIVERSITY OF POSTS AND TELECOM, 2004, Vol. 27 (3).

[10]Ye Wenchao. Discussion on Some Problems of Network Using NGN Technology. Telecommunications Science, 2003, Vol. (5). 